\title{
О СЕЧЕНИЯХ ПРОИЗВОДЯЩИХ РЯДОВ В ЗАДАЧАХ О РЕШЕТОЧНЫХ ПУТЯХ
}

\section{С. С. Ахтамова, В. Ю. Гришунов, А. П. Ляпин, С. А. Тихомиров}

(Статьл представлена членом редакционной коллегии С. М. Ситником)

Лесосибирский педагогический институт - филилал Сибирского федерального университета, г. Лесосибирск, 662544, Россия

ООО «Интермедиа»,

г. Санкт-Петербург, 191167, Россия

Сибирский федеральный университет, г. Красноярск, 660041, Россия

Ярославский государственный педагогический университет им. К. Д. Ушинского, г. Ярославль, 150000, Россия

E-mail: ahtamova-ss@mail.ru; vitaliqmetaliq@gmail.com; aplyapin@sfu-kras.ru; satikhomirov@mail.ru

Аннотация. В данной работе получена рекуррентная формула для сечений производящих рядов числа путей на целочисленной решетке и доказано, что сечения производящей функции для решеточных путей являются рациональными функциями (аналог теоремы Муавра).

Ключевые слова: разностные уравнения, решеточные пути, производящая функция, сечение.

Для цитирования: Ахтамова С. С., Гришунов В. Е., Ляпин А. П., Тихомиров С. А., 2020. О сечениях производящих рядов в задачах о решеточных путях. Прикладная математика \& Физика, 52(2): 146-151. DOI 10.18413/2687-0959-2020-52-2-146-151.

\section{ON SECTIONS OF GENERATING SERIES IN LATTICE PATH PROBLEMS}

\section{S. S. Akhtamova, V. Yu. Grishunov, A. P. Lyapin, S. A. Tikhomirov}

(Article submitted by a member of the editorial board C. M. Sitnik)

Lesosibirskij Pedagogical Institute - branch of Siberian Federal University,

Lesosibirsk, 662544, Russia

LLC «Intermedia»,

St. Petersburg, 191167, Russia

Siberian Federal University,

Krasnoyarsk, 660041, Russia

Yaroslavl State Pedagogical University named after K. D. Ushinsky,

Yaroslavl, 150000, Russia

E-mail: ahtamova-ss@mail.ru; vitaliqmetaliq@gmail.com; aplyapin@sfu-kras.ru; satikhomirov@mail.ru Received June 10, 2020

\begin{abstract}
In this paper a recurrence formula for the sections of the generating series of the number of paths on an integer lattice is obtained and it is proved that the sections of the generating function for the lattice paths are rational functions (an analogue of the Moivre theorem).

Key words: difference equations, lattice paths, generating function, section

For citation: Akhtamova S. S., Grishunov V. Y., Lyapin A. P., Tikhomirov S. A. 2020. On Sections Of Generating Series In Lattice Path Problems. Applied Mathematics \& Physics, 52(2): 146-151 (in Russian).
\end{abstract}

DOI 10.18413/2687-0959-2020-52-2-146-151.

1. Введение. В перечислительном комбинаторном анализе известна задача о перечислении решёточных путей: для набора векторов $\Delta=\left\{\alpha^{1}, \alpha^{2}, \ldots, \alpha^{N}\right\} \subset \mathbb{Z}^{n}$, требуется вычислить количество способов, которыми можно прийти из начала координат в точку $x \in \mathbb{Z}^{n}$, используя только шаги из набора $\Delta . \mathrm{K}$ числу известных путей на целочисленной решетке можно отнести пути Дика, Моцкина и Шредера (см. [Bousquet-Mélou, Petkovšek, 2000]). Отметим, что пути Дика связаны как со 
словами Дика, так и с диаграммами Юнга, деревьями и другими объектами перечислительного комбинаторного анализа (см. [Стенли, 2005], [Lyapin, Chandragiri, 2019]).

Если обозначить искомое число путей через $f(x)$, то известно (см. [6]), что $f(x)$ удовлетворяет рекуррентному соотношению

$$
f(x)-f\left(x-\alpha^{1}\right)-\ldots-f\left(x-\alpha^{N}\right)=0, x \in \mathbb{Z}^{n} .
$$

Мощным средством исследования свойств функции $f(x)$ являются производящие функции (см. [Стенли, 1990]), то есть функции вида

$$
F(z)=\sum_{x \in K} f(x) z^{x}
$$

которые позволяют эффективно использовать методы комплексного анализа для исследования свойств функции $f(x)$.

Абрахам Муавр в 1722 году доказал, что в одномерном случае степенной ряд $F(z)$ представляет собой рациональную функцию тогда и только тогда, когда его коэффициенты удовлетворяют рекуррентному соотношению (линейному разностному уравнению) с постоянными коэффициентами (cм. [Moivre, 1724]). А именно, коэффициенты разложения в степенной ряд рациональной функции

$$
F(z)=\frac{1}{c_{k} z^{k}+c_{k-1} z^{k-1}+\ldots+c_{0}}=\sum_{x=0}^{\infty} f(x) z^{x},
$$

где $c_{i} \in \mathbb{C}, i=0, \ldots, k$ - некоторые постоянные, удовлетворяют рекуррентному соотношению (разностному уравнению) для последовательности $\{f(x)\}_{x=0}^{\infty}$, все элементы которой, начиная с $k$-го, вычисляются через $k-1$ предыдущих:

$$
c_{k} f(x-k)+c_{k-1} f(x-k+1)+\ldots+c_{0} f(x)=0, x \geqslant k .
$$

Доказательство этого факта можно найти в [1], в главе, посвященной рациональным производящим функциям.

В общем случае, значения функции

$$
f(x)=\varphi(x), x=0, \ldots, k-1
$$

задаются произвольно, а задача (4)-(5) называется задачей Коши для разностного уравнения (4), а (5) - функцией начальных данных.

В многомерном случае ситуация гораздо сложнее и связана со свойствами и структурой конуса $K$ и, как следствие, различными видами разностных уравнений, описывающих число путей на целочисленной решетке. Отметим работу [Levy, Lessman, 1992], в которой для двумерного случая рассмотрены способы построения общих решений для некоторых видов разностных уравнений. В монографии [Даджион, Мерсеро, 1988] двумерные разностные уравнения использовались в теории цифровой обработки многомерных сигналов для конструирования цифровых рекурсивных фильтров. В случае двух переменных задача об устойчивости цифрового рекурсивного фильтра решена в работе [Tsikh, 1993]. В статье [Bousquet-Mélou, Petkovšek, 2000] многомерные разностные уравнения изучались с точки зрения применения к задачам перечислительного комбинаторного анализа. В ней сформулирована задача Коши для многомерного линейного разностного уравнения и доказана теорема существования и единственности решения этой задачи. В работе [Leinartas, 2007] приведена формула для решения задачи Коши с использованием понятия фундаментального решения. Ричард Стенли выделяет следующие классы производящих функций: D-финитные $\supset$ алгебраические つ рациональные, и рассматривает последние как «наиболее полезный» класс производящих функций (см. [Стенли, 1990]). В работе [Некрасова, 2014] исследуются производящие функции решений разностного уравнения в рациональных конусах целочисленной решетки. Для рядов Лорана с носителями в таких конусах определено понятие D-финитности и приведено достаточное условие, при котором из рациональности (алгебраичности, D-финитности) производящей функции начальных данных задачи Коши следует рациональность (алгебраичность, D-финитность) производящей функции решения. В данной работе доказан многомерный аналог теоремы Муавра (теорема 1), получена рекуррентная формула для сечений производящего ряда $F(z)$ и доказано, что сечения производящего ряда для решеточных путей являются рациональными функциями (следствие из теоремы 1).

2. Основные результаты. Рассмотрим набор векторов $\Delta=\left\{\alpha^{1}, \alpha^{2}, \ldots, \alpha^{N}\right\} \subset \mathbb{Z}_{\geqslant}^{n}$ и рассмотрим конус

$$
K=\left\{\alpha^{1} x_{1}+\ldots+\alpha^{N} x_{N}, x_{1}, \ldots, x_{N} \in \mathbb{Z}_{\geqslant}\right\} \subset \mathbb{Z}_{\geqslant}^{n}
$$


натянутый на вектора из набора $\Delta$ и лежащий в неотрицательном октанте. Известно (см., например, [Bousquet-Mélou, Petkovšek, 2000]), что производящий ряд для числа решеточных путей из начала координат в точку $x \in \mathbb{Z}_{\geqslant}^{n}$ сходится и имеет вид

$$
F(z)=\frac{1}{1-z_{1}^{\alpha_{1}^{1}} \cdots z_{n}^{\alpha_{n}^{1}}-\ldots-z_{1}^{\alpha_{1}^{N}} \ldots z_{n}^{\alpha_{n}^{N}}} .
$$

Определим сечение $\hat{F}^{k}(\hat{z}), k=0,1,2, \ldots$ производящего ряда $F(z)$ для числа решёточных путей с шагами из $\Delta$ следующим образом

$$
\hat{F}^{k}(\hat{z})=\sum_{\hat{z} \in \mathbb{Z}_{\geqslant}^{n-1}} f(k, \hat{x}) \hat{z}^{\hat{x}}
$$

где $\hat{z}=\left(z_{2}, z_{3}, \ldots, z_{n}\right), \hat{x}=\left(x_{2}, x_{3}, \ldots, x_{n}\right)$ (см. [Некрасова Т.И. 2014.], [Lipshitz, 1989]). В таких обозначениях будет иметь место запись $z=\left(z_{1}, \hat{z}\right)$ и $x=\left(x_{1}, \hat{z}\right)$. Заметим, что $\hat{F}^{0}(\hat{z})=F(0, \hat{z})$, а сами сечения представляют коэффициенты разложения функции $F(z)$ в степенной ряд по переменной $z_{1}$. Тогда справедлива следующая теорема.

Теорема 1. Сечения $\hat{F}^{k}(\hat{z})$ производящего ряда $F\left(z_{1}, \hat{z}\right)$ для решёточных путей с шагами из $\Delta$ удовлетворяют рекуррентному соотношению

$$
c_{k}(\hat{z}) \hat{F}^{n-k}(\hat{z})+c_{k-1}(\hat{z}) \hat{F}^{n-k+1}(\hat{z})+\cdots+c_{0}(\hat{z}) \hat{F}^{n}(\hat{z})=0
$$

с коэфбициентами $c_{l}(\hat{z})=\sum_{\alpha: \alpha_{1}^{i}=l} z_{2}^{\alpha_{2}^{i}} \ldots z_{n}^{\alpha_{n}^{i}}, l=0, \ldots, k$.

Доказательство. Сгруппируем слагаемые в знаменателе производящей функции (7) по степеням $z_{1}$ и разложим ее в степенной ряд по $z_{1}$ :

$$
F(z)=\frac{1}{c_{k}(\hat{z}) z_{1}^{k}+c_{k-1}(\hat{z}) z_{1}^{k-1}+\cdots+c_{0}(\hat{z}) z_{1}^{0}}=\sum_{n=0}^{\infty} \hat{F}^{n}(\hat{z}) z_{1}^{n},
$$

где $c_{l}(\hat{z})=\sum_{\alpha: \alpha_{1}^{i}=l} z_{2}^{\alpha_{2}^{i}} \ldots z_{n}^{\alpha_{n}^{i}}, l=0, \ldots, k$.

Домножим обе части этого равенства на знаменатель

$$
\left(c_{k}(\hat{z}) z_{1}^{k}+c_{k-1}(\hat{z}) z_{1}^{k-1}+\cdots+c_{0}(\hat{z}) z_{1}^{0}\right) \cdot \sum_{n=0}^{\infty} \hat{F}^{n}(\hat{z}) z_{1}^{n}=1
$$

и преобразуем левую часть

$$
\begin{aligned}
& \left(c_{k}(\hat{z}) z_{1}^{k}+c_{k-1}(\hat{z}) z_{1}^{k-1}+\cdots+c_{0}(\hat{z})\right) \cdot \sum_{n=0}^{\infty} \hat{F}^{n}(\hat{z}) z_{1}^{n}= \\
& =\sum_{n=k}^{\infty}\left(c_{k}(\hat{z}) \hat{F}^{n-k}(\hat{z})+c_{k-1}(\hat{z}) \hat{F}^{n-k+1}(\hat{z})+\cdots+c_{0}(\hat{z}) \hat{F}^{n}(\hat{z})\right) \cdot z_{1}^{n}+ \\
& \quad+\left(c_{k-1}(\hat{z}) \hat{F}^{0}(\hat{z})+c_{k-2}(\hat{z}) \hat{F}^{1}(\hat{z})+\cdots+c_{0} \hat{F}^{k-1}(\hat{z})\right) \cdot z_{1}^{k-1}+ \\
& \quad+\left(c_{k-2}(\hat{z}) \hat{F}^{0}(\hat{z})+\cdots+c_{0}(\hat{z}) \hat{F}^{k-2}(\hat{z})\right) \cdot z_{1}^{k-2}+\cdots+ \\
& \quad+\left(c_{1}(\hat{z}) \hat{F}^{0}(\hat{z})+c_{0}(\hat{z}) \hat{F}^{1}(\hat{z})\right) \cdot z_{1}+c_{0}(\hat{z}) \hat{F}^{0}(\hat{z}),
\end{aligned}
$$

затем, приравняв выражения при одинаковых степенях $z^{1}$, получим, что

$$
\left\{\begin{array}{l}
c_{0}(\hat{z}) \hat{F}^{0}(\hat{z})=1 \\
c_{1}(\hat{z}) \hat{F}^{0}(\hat{z})+c_{0}(\hat{z}) \hat{F}^{1}(\hat{z})=0 \\
\cdots \\
c_{k-2}(\hat{z}) \hat{F}^{0}(\hat{z})+\cdots+c_{0}(\hat{z}) \hat{F}^{k-2}(\hat{z})=0 \\
c_{k-1}(\hat{z}) \hat{F}^{0}(\hat{z})+c_{k-2}(\hat{z}) \hat{F}^{1}(\hat{z})+\cdots+c_{0} \hat{F}^{k-1}(\hat{z})=0
\end{array}\right.
$$

и для всех $n \geqslant k$ справедливо искомое рекуррентное соотношение:

$$
c_{k}(\hat{z}) \hat{F}^{n-k}(\hat{z})+c_{k-1}(\hat{z}) \hat{F}^{n-k+1}(\hat{z})+\cdots+c_{0}(\hat{z}) \hat{F}^{n}(\hat{z})=0
$$


Для доказательства обратного утверждения умножим левую часть данного равенства на $z^{k}$ и просуммируем по всем $n \geqslant k$. В итоге получим производящую функцию для решеточных путей. Теорема доказана.

Замечание 1. Отметим, что данная теорема справедлива для произвольной переменной $z_{j}$, где $j=1, \ldots, n$.

Замечание 2. По аналогии с задачей Кочи (4)-(5) для линейного разностного уравнения, можно сформулировать задачу Коши для уравнения (9), функция началъных данных $\hat{F}^{k}(\hat{z})=\hat{\Phi}^{k}(\hat{z})$, $k=0,1, \ldots, n-1$ которой может быть найдена из системы (10).

Замечание 3. Сечения производящего ряда $F(z)$ имеют и комбинаторный смысл, а именно функция $\hat{F}^{k}(\hat{z})$ являетсл производящей функцией для числа путей с иагами из набора $\Delta$, выходлщих из начала координат и оканчивающихся на гиперплоскости $x_{1}=k$.

Из теоремы 1 автоматически получается аналог теоремы Муавра для сечений производящих рядов числа путей на целочисленной решетке.

Следствие. Сечения $\hat{F}^{k}(\hat{z})$ производящего ряда $F\left(z_{1}, \hat{z}\right)$ для решёточных путей с шагами из набора $\Delta$ принадлежат $к$ классу рациональных функций.

3. Примеры. Пример 1. Рассмотрим набор векторов

$$
\Delta=\{(1,0,1),(2,2,2),(1,2,3),(3,1,2),(3,2,1),(2,1,2)\} .
$$

Производящая функция числа путей на целочисленной решетке будет иметь вид

$$
F\left(z_{1}, z_{2}, z_{3}\right)=\frac{1}{1-z_{1}\left(z_{3}+z_{2}^{2} z_{3}^{3}\right)-z_{1}^{2}\left(z_{2}^{2} z_{3}^{2}+z_{2} z_{3}^{2}\right)-z_{1}^{3}\left(z_{2} z_{3}^{2}+z_{2}^{2} z_{3}\right)},
$$

причем в обозначениях из теоремы $c_{0}\left(z_{2}, z_{3}\right)=1, c_{1}\left(z_{2}, z_{3}\right)=-z_{3}-z_{2}^{2} z_{3}^{3}, c_{2}\left(z_{2}, z_{3}\right)=-z_{2}^{2} z_{3}^{2}-z_{2} z_{3}^{2}$, $c_{3}\left(z_{2}, z_{3}\right)=-z_{2} z_{3}^{2}-z_{2}^{2} z_{3}$. Разностное уравнение имеет вид

$$
F^{n}\left(z_{2}, z_{3}\right)-\left(z_{3}+z_{2}^{2} z_{3}^{3}\right) F^{n-1}\left(z_{2}, z_{3}\right)-\left(z_{2}^{2} z_{3}^{2}+z_{2} z_{3}^{2}\right) F^{n-2}\left(z_{2}, z_{3}\right)-\left(z_{2} z_{3}^{2}+z_{2}^{2} z_{3}\right) F^{n-3}\left(z_{2}, z_{3}\right)=0 \text {. }
$$

Пример 2. Отметим связь сечений производящих рядов с многочленами Фибоначчи и Пелля (cм. [Luzon, Moron, 2010]).

Рассмотрим пути на целочисленной решетке, образованные набором шагов $\Delta=\{(1,1),(2,0)\}$. Функция $f\left(x_{1}, x_{2}\right)$ числа таких путей из начала координат в точку $\left(x_{1}, x_{2}\right)$ удовлетворяет разностному уравнению

$$
f\left(x_{1}, x_{2}\right)-f\left(x_{1}-1, x_{2}-1\right)-f\left(x_{1}-2, x_{2}\right)=0,
$$

а ее производящий ряд сходится и имеет вид $F\left(z_{1}, z_{2}\right)=\left(1-z_{1} z_{2}-z_{1}^{2}\right)^{-1}$. Обозначим $F^{n}\left(z_{2}\right)=$ $\sum_{x_{2} \geqslant 0} f\left(n, x_{2}\right) z_{2}^{x_{2}}$ - сечения производящего ряда. По теореме 1 такие сечения удовлетворяют рекур$x_{2} \geqslant 0$
рентному соотношению

$$
F^{n}\left(z_{2}\right)-z_{2} F^{n-1}\left(z_{2}\right)-F^{n-2}\left(z_{2}\right)=0
$$

с начальными данными $F^{0}\left(z_{2}\right)=1, F^{1}\left(z_{2}\right)=z_{2}$. Продолжая вычисления, получим известную последовательность многочленов Фибоначчи:

$$
\begin{aligned}
F^{2}\left(z_{2}\right) & =z_{2}^{2}+1 \\
F^{3}\left(z_{2}\right) & =z_{2}^{3}+2 z_{2}, \\
F^{4}\left(z_{2}\right) & =z_{2}^{4}+3 z_{2}^{2}+1, \\
F^{5}\left(z_{2}\right) & =z_{2}^{5}+4 z_{2}^{3}+3 z_{2}, \\
\ldots &
\end{aligned}
$$

Рассмотрим пути на целочисленной решетке, образованные набором шагов $\Delta=\{(1,1),(1,1)(2,0)\}$, для удобства будем считать, что шаг $(1,1)$ имеет кратность 2 или что существует два таких шага разного цвета. Функция $f\left(x_{1}, x_{2}\right)$ числа таких путей из начала координат в точку $\left(x_{1}, x_{2}\right)$ удовлетворяет разностному уравнению

$$
f\left(x_{1}, x_{2}\right)-2 f\left(x_{1}-1, x_{2}-1\right)-f\left(x_{1}-2, x_{2}\right)=0
$$

а ее производящий ряд сходится и имеет вид $F\left(z_{1}, z_{2}\right)=\left(1-2 z_{1} z_{2}-z_{1}^{2}\right)^{-1}$. По теореме 1 его сечения $F^{n}\left(z_{2}\right)$ удовлетворяют рекуррентному соотношению

$$
F^{n}\left(z_{2}\right)-2 z_{2} F^{n-1}\left(z_{2}\right)-F^{n-2}\left(z_{2}\right)=0
$$


с начальными данными $F^{0}\left(z_{2}\right)=1, F^{1}\left(z_{2}\right)=2 z_{2}$. Продолжая вычисления, получим известную последовательность многочленов Пелля:

$$
\begin{aligned}
F^{2}\left(z_{2}\right) & =4 z_{2}^{2}+1 \\
F^{3}\left(z_{2}\right) & =8 z_{2}^{3}+4 z_{2} \\
F^{4}\left(z_{2}\right) & =16 z_{2}^{4}+12 z_{2}^{2}+1 \\
\ldots &
\end{aligned}
$$

4. Заключение. Полученное рекуррентное соотношение для сечений производящих рядов числа путей на целочисленной решетке позволяет разработать компьютерный алгоритм для вычисления таких сечений.

\section{Список литературы}

1. Даджион Д., Мерсеро О. 1988. Цифровая обработка многомерных сигналов. Пер. с англ. М., Mup, 487. (Dudgeon D. E., Mersereau R. M. 1983. Multidimensional digital signal processing. Englewood Cliffs, NJ : Prentice-Hall, 400).

2. Лейнартас Е. К., Ляпин А. П. 2009. О рациональности многомерных возвратных степенных рядов. Журнал Сибирского федерального унивеситета. Математика и физика, 2(2): 449-455.

3. Некрасова Т. И. 2014. Об иерархии производящих функций решений многомерных разностных уравнений. Изв. Иркутского гос. ун-та. Сер. Математика, 9: 91-102.

4. Стенли Р. 2005. Перечислительная комбинаторика. Деревья, производящие функции и симметрические функции. М., Мир, 767.

5. Стенли Р. 1990. Перечислительная комбинаторика. М., Мир, 440.

6. Bousquet-Mélou M., Petkovšek M. 2000. Linear recurrences with constant coefficients: the multivariate case. Discrete Mathematics, 225: 51-75.

7. Leinartas E. K. 2007. Multiple Laurent series and fundamental solutions of linear difference equations, Siberian Math. J., 48(2): 268-272.

8. Levy H., Lessman F. 1992. Finite difference equations. Dover Publication, 278.

9. Lipshitz L. 1989. D-Finite power series. Journal of Algebra, 122: 353-373.

10. Luzon A., Moron M. A. 2010. Reccurence relations for polynomial sequences via Riordan matrices. Linear Algebra and its Applications, 433: 1422-1446. DOI:10.1016/j.laa.2010.05.021

11. Lyapin A. P., Chandragiri S. 2019. Generating functions for vector partitions and a basic recurrence relation. Journal of Difference Equations and Applications, 25(7): 1052-1061.

DOI: $10.1080 / 10236198.2019 .1649396$

12. Moivre A. 1724. De fractionibus algebraicis radicalitate immunibus ad fractiones simpliciores reducendis, deque summandis terminis quarumdam serierum aequali intervallo a se distantibus. Philosophical transactions, 176 .

13. Nekrasova T. I. 2015. On the Cauchy Problem for Multidimensional Difference Equations in Rational Cone. Journal of Siberian Federal University. Mathematics \& Physics, 8(2): 184-191.

14. Tsikh A. K. 1993. Conditions for absolute convergence of the Taylor coefficient series of a meromorphic function of two variables. Math. USSR-Sb, 74(2): 337-360.

\section{References}

1. Dudgeon D. E., Mersereau R. M. 1983. Multidimensional digital signal processing. Englewood Cliffs, NJ : Prentice-Hall, 400.

2. Leinartas E. K., Lyapin A. P. 2009. On the Rationality of Multidimentional Recusive Series. Journal of Siberian Federal University. Mathematics \& Physics, 2(2): 449-455 (in Russian). 
3. Nekrasova T. I. 2014. On the hierarchy of generating functions for solutions of multidimensional difference equations Izv. Irkutsk Gos. Univ. Ser. Matematika, 9: 91-103 (in Russian).

4. Stanley R. 1997. Enumerative combinatorics. Trees and the Composition of Generating Functions. Cambridge University Press, 600.

5. Stanley R. 1997. Enumerative combinatorics. Cambridge University Press, 326.

6. Bousquet-Mélou M., Petkovšek M. 2000. Linear recurrences with constant coefficients: the multivariate case. Discrete Mathematics, 225: 51-75.

7. Leinartas E.K. 2007. Multiple Laurent series and fundamental solutions of linear difference equations, Siberian Math. J., 48(2): 268-272.

8. Levy H., Lessman F. 1992. Finite difference equations. Dover Publication, 278.

9. Lipshitz L. 1989. D-Finite power series. Journal of Algebra, 122: 353-373.

10. Luzon A., Moron M. A. 2010. Reccurence relations for polynomial sequences via Riordan matrices. Linear Algebra and its Applications, 433: 1422-1446. DOI:10.1016/j.laa.2010.05.021

11. Lyapin A. P., Chandragiri S. 2019. Generating functions for vector partitions and a basic recurrence relation. Journal of Difference Equations and Applications, 25(7): 1052-1061.

DOI:10.1080/10236198.2019.1649396

12. Moivre A. 1724. De fractionibus algebraicis radicalitate immunibus ad fractiones simpliciores reducendis, deque summandis terminis quarumdam serierum aequali intervallo a se distantibus. Philosophical transactions, 176 .

13. Nekrasova T. I. 2015. On the Cauchy Problem for Multidimensional Difference Equations in Rational Cone. Journal of Siberian Federal University. Mathematics \& Physics, 8(2): 184-191.

14. Tsikh A. K. 1993. Conditions for absolute convergence of the Taylor coefficient series of a meromorphic function of two variables. Math. USSR-Sb, 74(2): 337-360.

Получена 10.06.2020

Ахтамова Светлана Станиславовна - кандидат педагогических наук, доцент, доцент кафедры высшей математики, информатики и естествознания Лесосибирского педагогического института филиала Сибирского федерального университета

ул. Победы, 42, г. Лесосибирск, Красноярский край, Россия, 662544

E-mail: ahtamova-ss@mail.ru

Гришунов Виталий Юрьевич - разработчик C\#/JavaScript, OOO «Интермедиа»

Невский пр-т., 2В, г. Санкт-Петербург, Ленинградская обл., Россия, 191167

E-mail: vitaliqmetaliq@gmail.com

Ляпин Александр Петрович - кандидат физико-математических наук, доцент базовой кафедры вычислительных и информационных технологий института математики и фундаментальной информатики Сибирского федерального университета

пр. Свободный, 79, г. Красноярск, Россия, 660041

E-mail: aplyapin@sfu-kras.ru

Тихомиров Сергей Александрович - кандидат физико-математических наук, доцент, доцент кафедры геометрии и алгебры физико-математического факультета Ярославского государственного педагогического университета

ул. Республиканская, 108/1, г. Ярославль, Россия, 150000

E-mail: satikhomirov@mail.ru 